\title{
ON SOME DESMIDS FROM KOSHI TAPPU WILDLIFE RESERVE, NEPAL
}

\author{
S.K. Rai and P.K. Misra* \\ Department of Botany, Post Graduate Campus \\ (Tribhuvan University) Biratnagar, Nepal \\ *Phycology Research Laboratory, Department of Botany \\ Lucknow University, Lucknow-226007, India
}

\begin{abstract}
The present work enumerates a total 26 desmids (Chlorophyta) from ponds and reservoirs in and around Koshi Tappu Wildlife Reserve, Nepal. They belong to 8 genera i.e. Closterium (4), Euastrum (4), Micrasterias (3), Cosmarium (7), Staurastrum (3), Onychonema (2), Hyalotheca (1), and Desmidium (2). Of these, 11 taxa are new records for Nepal. Genus Onychonema is described here for the first time from Nepal.
\end{abstract}

Key words: Algae, chlorophyta, Desmids, Koshi Tappu, Nepal.

\section{INTRODUCTION}

The first contribution on the desmid flora of Nepal was made by Hirano (1955) who had reported 79 taxa from the Himalayan region of Western Development Region (Watanabe 1982). Later on, considerable reports have been presented by Hirano (1963, 1969, 1984), Förster (1965), Kusel-fetzmann (1969), Hickel (1973), Ichimura and Kasai (1982), Watanabe (1982, 1995), Shrestha and Manandhar (1983), Nakanishi (1986), Bando et al. (1989), and Habib and Chaturvedi $(1995,1997)$. These works seem to be sporadic and most of them were concentrated to the high altitude regions. Since then, there is no any report on the desmids published for the country within this decade. Bando et al. (1989) have considered that the southeastern part of Asia is the most important region for the origin and distribution of desmids. Therefore, the terai region of Nepal, due to its hot and humid climate, favour luxuriant growth of desmids. But, literature revealed that this area has not been explored so far properly. Thus, it was felt desirable to explore the algae from Koshi Tappu area.

Koshi Tappu Wildlife Reserve (Lat. 26 $34^{\circ}$ $26^{\circ} 45^{\prime} \mathrm{N}$, Long. $86^{\circ} 55^{\prime}-87^{\circ} 05^{\prime} \mathrm{E}$; elevation $70-120$ $\mathrm{m}$ amsl; area $175 \mathrm{~km}^{2}$ ) lies in the flood plain of Sapta Koshi river spreading in Sunsari and Saptari districts, more or less in rectangular shape. It was established as a protected area in 1976 and declared a Ramsar site in 1987 to conserve many endangered species including Wild buffalo (Bubalus arnee), Gharial (Gavialis gangeticus) and Gangetic dolphin (Platanista gangetica). The Sapta Koshi river has formed many permanent ponds and marshy lands along its length and seepage stream along the eastern embankment. The area has subtropical climate with maximum temperature in May and maximum rainfall in July. 
This communication describes the morphology and taxonomy of 26 desmids belonging to 8 genera of desmidiaceae from different freshwater lentic environments of Koshi Tappu Wildlife Reserve and its adjoining area and also records their distribution in other parts in Nepal. Of these, 11 taxa viz., Euastrum platycerum Reinsch, Micrasterias foliacea Bail., Cosmarium lundelli Delp. var. ellipticum W. et G.S. West f. minus Prescott, C. pardalis Cohn., C. subspeciosum Nordst. var. validius Nordst., C. zonatum Lund. var. javanicum (Gutw.) Krieg., Staurastrum leptocladum Nordst. var. cornutum Wille, St. sexangulare Lund. var. productum Nordst., Onychonema filiforme (Ehr. ex Ralfs) Roy et Biss., O. leave Nordst. var. latum W. et G.S. West, and Desmidium baileyi (Ralfs) Nordst. var. baileyi f. tetragonum Nordst. are new records for Nepal. Onychonema is a genus described here for the first time for the country. As no work exists on the desmids hitherto, all these taxa are new record for this area.

\begin{tabular}{|c|c|c|}
\hline $\begin{array}{c}\text { Sample } \\
\text { No. }\end{array}$ & $\begin{array}{c}\text { Date of } \\
\text { collection }\end{array}$ & Locality \\
\hline EN 96 & 21.12 .2002 & $\begin{array}{l}\text { Grass green, thick layered } \\
\text { assemblage on shallow } \\
\text { water near Hand-pump, } \\
\text { beside a temple in the } \\
\text { Reserve. }\end{array}$ \\
\hline EN 101 & 21.12 .2002 & $\begin{array}{l}\text { Planktonic in the south- } \\
\text { west shallow edge of } \\
\text { Titrigachi pond. }\end{array}$ \\
\hline EN 128 & 29.03 .2003 & $\begin{array}{l}\text { Epiphytic on the root of } \\
\text { Eichhornia crassipes and } \\
\text { Pistia stratiotes on the edge } \\
\text { of Koshi Barrage } \\
\text { Reservoir, just north of } \\
\text { Mahendra Highway, west } \\
\text { from Bhantabari. }\end{array}$ \\
\hline EN 129 & 29.03 .2003 & $\begin{array}{l}\text { Planktonic in the south-east } \\
\text { shallow edge of Titrigachi } \\
\text { pond, along the north side } \\
\text { of embankment. }\end{array}$ \\
\hline
\end{tabular}

\section{MATERIALS AND METHODS}

Epiphytic forms were collected by squeezing out the root of aquatic macrophytes and planktonic forms with the help of plankton net (mesh size 50 $\mu \mathrm{m})$. Samples were tagged, labelled and then preserved with $4 \%$ formaldehyde solution. Morphotaxonomic observation and photomicrography were done with the help of Nikon Labophot Microscope in the Phycology Research Laboratory, Department of Botany, Lucknow University, India. Identification was based on illustration and dimension of the literature mentioned below the name of each taxon.

\section{SYSTEMATIC DESCRIPTIONS}

\section{Family: Desmidiaceae}

\section{Genus: Closterium Nitzsch 1817}

Closterium acerosum (Schrank) Ehr. ex Ralfs (Pl. 1, Fig. 8)

Scott, A.M. and G.W. Prescott 1961, P. 9, Pl. 3, Fig. 1; Gerrath, J.F. and D.M. John 1988, P. 195, Pl. 9, Fig. 1; Prasad, B.N. and P.K. Misra 1992, P. 97, Pl. 16, Fig. 15.

Cells 145-260 $\mu \mathrm{m}$ long, 30-32 $\mu \mathrm{m}$ broad, nearly straight or slightly curved, narrowly fusiform; outer margin curved with 30-36 degrees arc, inner margin straight, gradually tapering towards the rounded-truncate or sub-acute ends, apices 4.5-6 $\mu \mathrm{m}$ broad; cell wall smooth; chloroplast 3-5 ridged with 5-6 pyrenoids in a median series in each semicell.

\section{Sample No.: EN 96}

Distribution in Nepal: Rocky wall at Pangka along Dudh Koshi river, $4600 \mathrm{~m}$; shallow flow at Longponga at the end of Ngozumba glacier, 4650 $\mathrm{m}$; and shallow pool at the shore of Dudh pokhari lake at Gokyu, $4750 \mathrm{~m}$ (Watanabe 1982); roadside ditches at Mahendranagar road and a puddle near 
Mahendranagar Bazaar (Habib and Chaturvedi 1995, 1997)

Closterium dianae Ehr. ex Ralfs var. dianae (Pl. 2, Fig. 5)

Kouwets, F.A.C. 1987, P. 201, Pl. 2, Figs. 23-28; Flint, E.A. and D.B. Williamson 1998, P. 75, Pl. 2, Fig. 7

Cells $330 \mu \mathrm{m}$ long, $26.5 \mu \mathrm{m}$ broad; outer margin curved with 108 degree arc, inner slightly tumid or inflated in the middle, gradually tapering towards the obtusely rounded ends, apices 2-3 $\mu \mathrm{m}$ broad; cell wall smooth; chloroplast 3-4 ridged with 6-8 pyrenoids in a median series in each semicell.

Remarks: Our specimen has slightly larger dimension.

Sample No.: EN 129

Distribution in Nepal: Shallow streamlet near Begnash lake and shallow flow along rice field at Hetauda, 500 m (Watanabe 1982)

Closterium ehrenbergii Menegh. ex Ralfs (Pl. 1, Fig. 6)

Scott, A.M. and G.W. Prescott 1961, P. 11, Pl. 2, Fig. 2; Kouwets, F.A.C. 1987, P. 203, Pl. 2, Fig. 22; Gerrath, J.F. and D.M. John 1988, P. 197, Pl. 6, Figs. 1-2.

Cells $380 \mu \mathrm{m}$ long, $59 \mu \mathrm{m}$ broad, stout, moderately curved; outer margin with 100-125 degrees of arc, inner margin concave, slightly inflated in the middle, cells gradually tapering to the obtusely rounded ends; apices 8-10 $\mu \mathrm{m}$ broad; cell wall smooth, colourless; chloroplast 6-8 ridged with numerous scattered pyrenoids.

Sample No.: EN 101

Distribution in Nepal: Streamlet between Naubise and Mugling, $500 \mathrm{~m}$, Dhading and shallow flow along rice field at Hetauda, $500 \mathrm{~m}$ (Watanabe 1982); rice field at Baudha, $1325 \mathrm{~m}$; pool near
Pashupatinath, $1320 \mathrm{~m}$; ring road near Bansbari, $1320 \mathrm{~m}$, Kathmandu; pond at Dulari, $50 \mathrm{~m}$, Morang; pond at Laghula, $5 \mathrm{~km}$ west of Itahari, 40 $\mathrm{m}$; and pond at Birganj, $40 \mathrm{~m}$, Parsa (Ichimura and Kasai 1982); ringroad ditches at $2 \mathrm{~km}$ north of Chabahil, Kathmandu (Bando et al. 1989); pond near Mahendranagar Bazar, Kanchanpur (Habib and Chaturvedi 1997)

Closterium setaceum Ehr. ex Ralfs (Pl. 1, Fig. 10)

Scott, A.M. and G.W. Prescott 1961, P. 13, Pl. 1, Fig. 21; Kouwets, F.A.C. 1987, P. 207, Pl. 5, Figs. 2-3; Gerrath, J.E. and D.M. John 1988, P. 204, Pl. 9, Fig. 4.

Cells $380 \mu \mathrm{m}$ long, 13.5-15 $\mu \mathrm{m}$ broad, almost straight, median part fusiform-lanceolate with convex margins, tapering into long setaceous processes with rounded ends, apices 2-3 $\mu \mathrm{m}$ broad; cell wall longitudinally striated; chloroplast with 45 pyrenoids in a median series

Sample No.: EN 129

Distribution in Nepal: Pond at Dillibazar, 1300m, Kathmandu (Hirano 1963); small pond south of Rara lake, 3030 m, Mugu (Watanabe 1995)

\section{Genus: Euastrum Ehrenberg 1832}

Euastrum bidentatum Näg. (P1. 2, Fig. 4)

Capdevielle, P. and A. Coute' 1980, P. 880, Pl. 2 , Fig. 20; Alfinito, S. and B. Fumanti 1980, P. 880, Pl. 2, Fig. 20

Cells $44 \mu \mathrm{m}$ long, $30 \mu \mathrm{m}$ broad, deeply constricted, sinus slightly dilated at the extremity; isthmus narrow, 6-7 $\mu \mathrm{m}$ wide; semicells 3-lobed; polar lobe $19 \mu \mathrm{m}$ broad with deep median incision, apical angles with a short spine; lateral lobes bilated, marginal spines not distinct; semicells with 5 protuberances, one large just above the isthmus, one on each lateral lobe and one on each side of apical notch in the polar lobe. 
Sample No.: EN 101

Distribution in Nepal: Pheriche, 4200m, Khumbu as E. bidentatum Näg. var. speciosum Boldt ex Schmidle (Syn. E. elegans var. speciosum Boldt) (Förster, 1965)

Euastrum insulare (Wittr.) Roy (P1. 1, Fig. 14)

Capdevielle, P. and A. Coute' 1980, P. 880, P1. 2, Fig. 19; Kouwets, F.A.C. 1987, P. 216, Pl. 7, Fig. 21

Cells 21-22 $\mu \mathrm{m}$ long, $14.8 \mu \mathrm{m}$ broad, deeply constricted, sinus narrowly linear; isthmus 4.5-5 $\mu \mathrm{m}$ wide; semicells truncate, 3 lobed; polar lobe 10 $\mu \mathrm{m}$ broad, short and broadly truncate with shallow median notch, apical angles rounded without marginal spine; lateral lobes broadly rounded; protuberances are not distinct.

Sample No.: EN 101

Distribution in Nepal: A stream at Mewa Valley (Hirano 1984)

\section{Euastrum platycerum Reinsch (P1. 2, Fig. 7)}

Scott, A.M. and G.W. Prescott 1961, P. 33, P1. 60, Fig. 4; Nurul Islam, A.K.M. 1970, P. 917, P1. 17, Figs. 5-6; Gerrath, J.F. and D.M. John 1988, P. 210, P1. 8, Figs. 13-14.

Cells 40-42 $\mu \mathrm{m}$ long, 37-37.5 $\mu \mathrm{m}$ broad, deeply constricted, sinus narrow, slightly dilated and widely open out; isthmus $8-10 \mu \mathrm{m}$ wide; semicells 3 lobed; polar lobes 12.5-13 $\mu \mathrm{m}$ broad, truncate without median constriction, broadly rounded angles with 2 small marginal spines; lateral lobes broadly rounded with 5 small marginal spines; semicells with a rounded central protuberance just above the isthmus.

Sample No.: EN 101

Distribution in Nepal: New record for Nepal.

Euastrum spinulosum Delp. (P1. 2, Fig. 6)

Scott, A.M. and G.W. Prescott 1961, P. 40, P1. 10, Fig. 3; Nurul Islam, A.K.M. and A.K. Yusuf
Haroon 1980, P. 568, P1. 22, Fig. 356; Yacubson, S. 1980, P. 301, Pl. 11, Fig. 132.

Cells $52 \mu \mathrm{m}$ long, $47 \mu \mathrm{m}$ broad, deeply constricted, sinus narrow, linear; isthmus 11.5-13 $\mu \mathrm{m}$ wide; semicells 5 lobed; polar lobe 17-18 $\mu \mathrm{m}$ broad, broadly truncate with shallow median notch, broadly rounded angles each with 3 small marginal spines; lateral lobes broadly rounded with 5-6 small marginal spines; cell wall granulate in the lobes; each semicell with a rounded, central protuberance.

\section{Sample No.: EN 101}

Distribution in Nepal: Ankhu Khola, $640 \mathrm{~m}$ and Luitel Bhanjyang, 770 m, Gorkha (Hirano 1955 as var. inermius); ditches at Chabahil, Kathmandu (Bando et al. 1989 as var. javanicum)

Genus: Micrasterias C.A. Agardh 1827

\section{Micrasterias foliacea Bailey (P1. 1, Fig. 2)}

Turner, W.B. 1892, P. 24, Pl. 6, Figs. 12-15; Scott, A.M. and G.W. Prescott 1961, P. 48, Pl. 20, Fig. 4; Nurul Islam, A.K.M. 1970, P. 919, Pl. 9, Figs. 5-6; P1. 12, Figs. 4-5.

Cells $60-70 \mu \mathrm{m}$ long, $80 \mu \mathrm{m}$ broad, united into filaments by inter-locking of polar lobes, outline rectangular, deeply constricted, sinus narrowly linear; isthmus 14-18 $\mu \mathrm{m}$ wide; semicells 5 lobed; polar lobes 23-26 $\mu \mathrm{m}$ long, 38-40 $\mu \mathrm{m}$ wide, exserted, sub-parallel base and expanded but excavated upper part, 2 unequal and asymmetrical spines at the base of excavation; lateral lobes with unequal lobelets, each lobelet terminating into truncate-emerginate apices; cell wall smooth.

Sample No.: EN 101

Distribution in Nepal: New record for Nepal.

Micrasterias mahabuleshwarensis Hobson (P1. 2, Fig. 8)

Nurul Islam, A.K.M. 1970, P. 920, Pl. 9, Fig. 3; Prasad, B.N. and P.K. Misra 1992, P. 142, Pl. 20, Fig. 7 
Cells $120 \mu \mathrm{m}$ long, $104 \mu \mathrm{m}$ broad, deeply constricted, sinus open with acuminate extremity; isthmus 20-21 $\mu \mathrm{m}$ wide; semicells 3-lobed in 3 symmetric planes with wide incision; polar lobes nearly subquadrate with two pairs of diverging, asymmetrical, denticulated processes; lateral lobes divided into 2 attenuated and denticulated processes by a wide, acute incision; all processes ends into 3 or 4 small spines.

Sample No.: EN 129

Distribution in Nepal: Taudaha, $1350 \mathrm{~m}$, Kathmandu (Bando et al. 1989)

Micrasterias pinnatifida (Kütz.) Ralfs (P1. 2, Fig. 11)

Scott, A.M. and G.W. Prescott 1961, P. 51, Pl. 12, Fig. 6; P1. 14, Figs. 17-18; Nurul Islam, A.K.M. 1970, P. 920, Pl. 10, Figs. 3-7; Nurul Islam, A.K.M. and A.K. Yusuf Haroon 1980, P. 572, P1. 14, Figs. 191-192, 199.

Cells $56 \mu \mathrm{m}$ long, $60 \mu \mathrm{m}$ broad, deeply constricted, sinus linear but open out; isthmus 10$11 \mu \mathrm{m}$ wide; semicells 3 lobed with deep and rounded incision; polar lobe $13 \mu \mathrm{m}$ long, $38 \mu \mathrm{m}$ broad, nearly triangular, with shallow median notch; lateral lobes horizontal, semifusiform; all lobes acuminated toward the end with bifid spines; spines 4-5 $\mu \mathrm{m}$ long; cell wall minutely punctuate.

Sample No.: EN 101

Distribution in Nepal: A pond at Luitel Bhanjyang, 770 m, Gorkha (Hirano 1955)

Genus: Cosmarium Corda 1834

\section{Cosmarium javanicum Nordst. (P1. 2, Fig. 1)}

Nurul Islam, A.K.M. and A.K. Yusuf Haroon 1980, P. 576, P1. 11, Figs. 156-157; Pl. 13, fig. 179; Bando, T. et al. 1989, P. 16, Fig. $7 \mathrm{f}$
Cells $167 \mu \mathrm{m}$ long, $80 \mu \mathrm{m}$ broad, moderately constricted, sinus narrow, dilated at the apex; isthmus $40 \mu \mathrm{m}$ wide; semicells truncatepyramidate, basal angles rounded, sides convex, truncately rounded ends; cell wall distinctly punctate.

\section{Sample No.: EN 101}

Distribution in Nepal: A pond at Luitel Bhanjyang, 770 m, Gorkha (Hirano 1955); Taudaha, 1350 m, Kathmandu (Bando et al. 1989); Sharada river near

Mahendranagar, Kanchanpur (Habib and Chaturvedi 1995)

Cosmarium lundelli Delp. var. ellipticum W. et G.S. West (P1. 1, Fig. 11)

Scott, A.M. and G.W. Prescott 1961, P. 61, P1. 25, Fig. 8; Prasad, B.N. and P.K. Misra 1992, P. 164, P1. 22, Fig. 23; Nurul Islam, A.K.M. and H.M. Irfanullah 1999, P. 93, P1. 1, Figs. 6-7.

Cells $57 \mu \mathrm{m}$ long, $42.5 \mu \mathrm{m}$ broad, deeply constricted, sinus narrowly linear, closed; isthmus $16 \mu \mathrm{m}$ wide; semicells sub-semicircular with rounded basal angle and broadly rounded ends; cell wall punctate; chloroplast axile with two pyrenoids.

Sample No.: EN 101

Distribution in Nepal: A pond at Luitel Bhanjyang, 770 m, Gorkha (Hirano 1955); pond at Patandhoka, 1300 m, Lalitpur (Hirano 1963); pond near Nepal forest, Mahendranagar, Kanchanpur (Habib and Chaturvedi 1997)

Cosmarium lundelli Delp. var. ellipticum W. et G.S. West f. minus Prescott (P1. 2, Fig. 13)

Bharati, S.G. and G.R. Hegde 1982, P. 744, P1. 1, Fig. 6; Prasad, B.N. and P.K. Misra 1992, P. 164, P1. 22, Fig. 23. 
Cells $47 \mu \mathrm{m}$ long, $41.5 \mu \mathrm{m}$ broad, deeply constricted, sinus narrowly linear, slightly open out; isthmus $19.5 \mu \mathrm{m}$ wide; semicells subsemicircular to sub-pyramidate with broadly rounded and shallow median constricted ends; cell wall punctate; chloroplast axile with two pyrenoids.

Sample No.: EN 101

Distribution in Nepal: New record for Nepal.

Cosmarium obsoletum (Hantz.) Reinsch (P1. 2, Fig. 3)

Scott, A.M. and G.W. Prescott 1961, P. 63, Pl. 26, Fig. 1; Bharati, S.G. and G.R. Hegde 1982, P. 746, P1. 1, Fig. 5; Kouwets, F.A.C. 1987, P. 226, Pl. 11, Fig. 15

Cells $43 \mu \mathrm{m}$ long, $50 \mu \mathrm{m}$ broad, transversely elliptic, deeply constricted, sinus narrowly linear with dilated apex and slightly open out; isthmus $22.5 \mu \mathrm{m}$ wide; semicells depressed-semicircular, basal angles submamillate and slightly thickened, slightly flattened ends; cell wall punctate; chloroplast axile with two pyrenoids.

Sample No.: EN 101

Distribution in Nepal: A pond at Luitel Bhanjyang, 770 m, Gorkha (Hirano 1955)

Cosmarium pardalis Cohn. (Pl. 2, Fig. 2)

Scott, A.M. and G.W. Prescott 1961, P. 64, Pl. 29, Figs. 1-2; Nurul Islam, A.K.M. 1970, P. 926, Pl. 13, Fig. 7.

Cells 85-90 $\mu \mathrm{m}$ long, $74 \mu \mathrm{m}$ broad, deeply constricted, sinus gradually opening from a dilated extremity; isthmus $35 \mu \mathrm{m}$ wide; semicells reniform; cell wall granulate, granules arranged in horizontal and indistinct vertical series; chloroplast axile with 2 pyrenoids.
Sample No.: EN 128

Distribution in Nepal: New record for Nepal.

Cosmarium subspeciosum Nordst. var. validius Nordst. (P1. 1, Fig. 12)

Yacubson, S. 1980, P. 300, Pl. 10, Fig. 114; Bharati, S.G. and G.R. Hegde 1982, P. 752, P1. 9, Fig. 1; Sahin, B. 2005, P. 409, Fig. 14.

Cells $45 \mu \mathrm{m}$ long, $32.5 \mu \mathrm{m}$ broad, deeply constricted, sinus narrowly linear and close; isthmus 11-12 $\mu \mathrm{m}$ wide; semicells sub-rectangular or sub-pyramidate with rounded angles, convex sides and very gradually attenuated towards broadly truncate ends, margins with 4 apical and 7 lateral crenations; cell wall granulate; chloroplast axile with 2 pyrenoids.

Sample No.: EN 101

Distribution in Nepal: New record for Nepal.

Cosmarium zonatum Lund. var. javanicum (Gutw.) Krieg. (P1. 1, Fig. 13)

Scott, A.M. and G.W. Prescott 1961, P. 73, Pl. 28, Fig. 5 (as C. zonatum); Nurul Islam, A.K.M. and A.K. Yusuf Haroon 1980, P. 582, P1. 12, Figs. 162-163.

Cells $37.5 \mu \mathrm{m}$ long, $23.5 \mu \mathrm{m}$ broad, subhexagonal, constricted, sinus linear with widely open out; isthmus 7.5-8 $\mu \mathrm{m}$ wide; semicells with broadly rounded basal angles, converging and retuse upper lateral margins, ends faintly retuse with rounded angles; cell wall thick and smooth; chloroplast with one pyrenoid.

Sample No.: EN 129

Distribution in Nepal: New record for Nepal.

Genus: Staurastrum Meyen 1829 
Staurastrum leptocladum Nordst. var. cornutum Wille (P1. 2, Fig. 12)

Turner, W.B. 1892, P. 123, Pl. 14, Figs. 12, 16; Nurul Islam, A.K.M. and A.K. Yusuf Haroon 1980, P. 590, Pl. 17, Fig. 243; Therezien, Y. 1985, P. 552, P1. 25, Fig. 3.

Cells $36 \mu \mathrm{m}$ long (without spines), 10-15 $\mu \mathrm{m}$ (without processes) to 55-67 $\mu \mathrm{m}$ (with processes) broad, constricted, sinus open out; isthmus 6-7 $\mu \mathrm{m}$ wide; semicells slightly elongated at the base, broadening upward, dorsal margin slightly convex with a pair of divergent spines; processes long, slightly convergent, crenulate margin with 2 terminal spines; chloroplast with one pyrenoid.

Sample No.: EN 129

Distribution in Nepal: New record for Nepal.

Staurastrum sexangulare Lund. var. productum Nordst. (P1. 2, Fig. 9)

Scott, A.M. and G.W. Prescott 1961, P. 107, P1. 46, Figs. 3-4; Nurul Islam, A.K.M. and A.K. Yusuf Haroon 1980, P. 592, Pl. 19, Fig. 289.

Cells 17.5-30 $\mu \mathrm{m}$ (without processes) and $55 \mu \mathrm{m}$ (with processes) broad; semicells with 5 large processes, each large process provided with a small process at its lower side at the base, each process terminate into 3-5 divergent spines.

Sample No.: EN 129

Distribution in Nepal: New record for Nepal.

Staurastrum sexcostatum Bréb. ex Ralfs var. productum (W. West) G.S. West (Pl. 2, Fig. 10)

Kouwets, F.A.C. 1987, P. 251, P1. 19, Fig. 7; Kim, H.S. 1996, P. 536, Pl. 7, Fig. 68.

Cells $39.5 \mu \mathrm{m}$ long, 42-42.5 $\mu \mathrm{m}$ broad (with processes), shallow constriction with an acute notch; isthmus $15 \mu \mathrm{m}$ wide; semicells nearly hexagonal to cup shaped, dorsal margin straight to slightly convex; processes 6 , stout, each 7.5-9 $\mu \mathrm{m}$ long, $6.3 \mu \mathrm{m}$ broad at the base, slightly convergent with 3 marginal crenulations and 3 short terminal spines; chloroplasts with 1 pyrenoid.

Sample No.: EN 129

Distribution in Nepal: A pond at Luitel Bhanjyang, 770 m, Gorkha (Hirano 1955)

Genus: Onychonema Wallich 1860 (New genus)

Onychonema filiforme (Ehr. ex Ralfs) Roy et Biss. (Pl. 1, Fig. 7)

Tiffany, L.H. and M.E. Britton 1952, P. 201, Pl. 56, Figs. 621-623; Flint, E.A. and D.B. Williamson 1998, P. 95, Pl. 10, Fig. 7

Cells $15.6 \mu \mathrm{m}$ long (without spines), $15 \mu \mathrm{m}$ broad, united into long twisted filament by the processes, deeply constricted, sinus sublinear with acute apex and wide open out; isthmus 4.5-5 $\mu \mathrm{m}$ wide; semicells broadly elliptic or sub-reniform, dorsal and lateral margins broadly rounded, ventral margin almost straight; processes long, divergent asymmetrical with slight swelling at the ends; chloroplast axial with 1 pyrenoid.

Sample No.: EN 129

Distribution in Nepal: New record for Nepal.

Onychonema laeve Nordst. var. latum W. et G.S. West (Pl. 1, Fig. 5)

Turner, W.B. 1892, P. 139, Pl. 17, Fig. 15; Tiffany, L.H. and M.E. Britton 1952, P. 201, Pl. 56, Fig. 624; Scott, A.M. and G.W. Prescott 1961, P. 121, P1. 60, Fig. 13.

Cells 15-16 $\mu \mathrm{m}$ long, $19 \mu \mathrm{m}$ (without spines) to 22.5-23 $\mu \mathrm{m}$ (with spines) broad, deeply 
constricted, sinus open, united into filaments, filament embedded in gelatinous sheath; isthmus 4$4.5 \mu \mathrm{m}$ wide; semicells transversely elliptic; apex with 2 divergent processes, lateral angles with 1 incurved spine; chloroplast axial with 1 pyrenoid.

Sample No.: EN 129

Distribution in Nepal: New record for Nepal.

Genus: Hyalotheca Ehrenberg 1841

Hyalotheca dissiliens (J.E. Smith) ex Bréb. in Ralfs (P1. 1, Fig. 3)

Tiffany, L.H. and M.E. Britton 1952, P. 204, P1. 56, Fig. 631; Kouwets, F.A.C. 1987, P. 256, P1. 21, Figs. 4-5.

Cells $16 \mu \mathrm{m}$ long, $20 \mu \mathrm{m}$ broad, cylindric to discoidal, constricted at the joints, sinus very slightly concave, united into long filament by truncate apices, filaments embedded in a gelatinous sheath; chloroplast axial with 1 pyrenoid in each semicell.

Sample No.: EN 101

Distribution in Nepal: A pond at Pisang, $3100 \mathrm{~m}$, Manang (Hirano 1955); Langtang valley, $3700 \mathrm{~m}$, Rasuwa (Hirano 1969); a small pond south of Rara lake, 3030 m, Mugu (Watanabe 1995)

Genus: Desmidium C.A. Agardh 1824

Desmidium baileyi (Ralfs) Nordst. var. baileyi f. tetragonum Nordst. (Pl. 1, Fig. 9)

Scott, A.M. and G.W. Prescott 1961, P. 124, Pl. 62, Figs. 8-9; Nurul Islam, A.K.M. and H.M. Irfanullah 1999, P. 120, Pl. 1, Fig. 1.
Cells 15-16.5 $\mu \mathrm{m}$ long, 20-20.5 $\mu \mathrm{m}$ broad, rectangular with parallel lateral margins and broad apices with deep and semi-elliptic depression, median constriction reduced into undulation, united into a straight filament without gelatinous sheath; isthmus $17.5 \mu \mathrm{m}$ wide; cell wall smooth; chloroplast axial.

Sample No.: EN 101

Distribution in Nepal: New record for Nepal.

Desmidium swartzii (Ag.) Ag. ex Ralfs (Pl. 1, Figs. 1 and 4)

Scott, A.M. and G.W. Prescott 1961, P. 125, Pl. 63, Fig. 8; Kouwets, F.A.C. 1987, P. 256, P1. 21, Fig. 2; Prasad, B.N. and P.K. Misra 1992, P. 204, P1. 26, Fig. 15.

Cells 14.2-16.2 $\mu \mathrm{m}$ long, $25 \mu \mathrm{m}$ broad, narrowly rectangular, moderately constricted, sinus linear but widely open out, united into spirally twisted filaments; isthmus 19.7-20 $\mu \mathrm{m}$ wide; semicells narrowly oblong, lateral margins obliquely truncate with upper angle protruded toward the apex, apex slightly straight without depression; cell wall smooth; chloroplast axial.

Sample No.: EN 129

Distribution in Nepal: Ringroad ditches at $2 \mathrm{~km}$ north of Chabahil, Kathmandu (Bando et al. 1989); a small pond south of Rara lake, 3030 m, Mugu (Watanabe 1995); a pond at Indo-Nepal border, Mahendranagar, Kanchanpur (Habib and Chaturvedi 1997) 

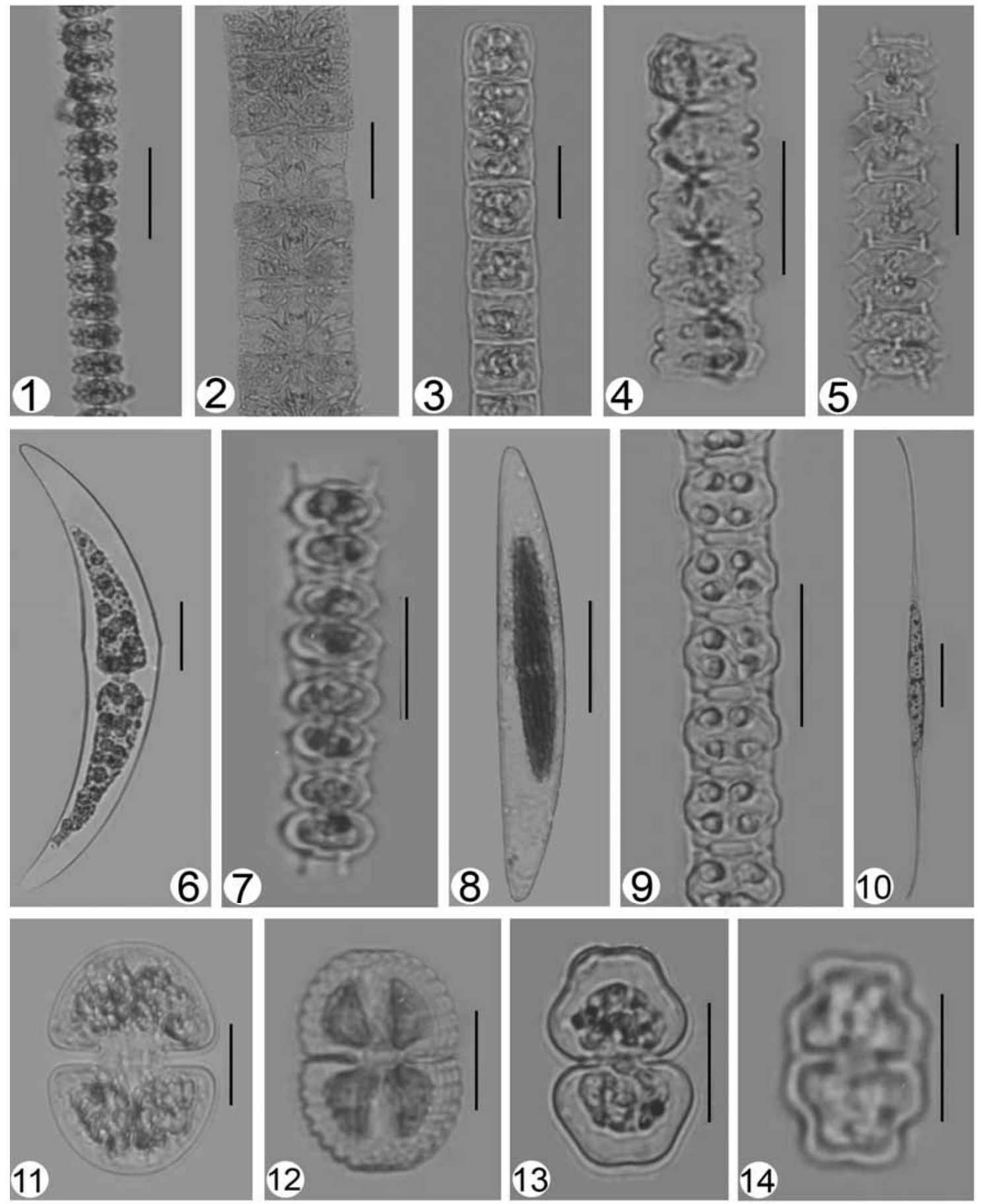

Plate 1. (Figs. 1\&4. Desmidium swartzii (Ag.) Ag. ex Ralfs, Fig. 2. Micrasterias foliacea Bailey, Fig. 3. Hyalotheca dissiliens (J.E. Smith) ex Bréb. in Ralfs, Fig. 5. Onychonema laeve Nordst. var. latum W. et G.S. West, Fig. 6. Closterium ehrenbergii Menegh. ex Ralfs, Fig. 7. Onychonema filiforme (Ehr. ex Ralfs) Roy et Biss., Fig. 8. Closterium acerosum (Schrank) Ehr. ex Ralfs, Fig. 9. Desmidium baileyi (Ralfs) Nordst. var. baileyi f. tetragonum Nordst., Fig. 10. Closterium setaceum Ehr. ex Ralfs, Fig. 11. Cosmarium lundelli Delp. var. ellipticum W. et G.S. West, Fig. 12. Cosmarium subspeciosum Nordst. var. validius Nordst., Fig. 13. Cosmarium zonatum Lund. var. javanicum (Gutw.) Krieg., Fig. 14. Euastrum insulare (Wittr.) Roy)

(Scales in the figs. 3, 5, 7, 11, 12, $13=20 \mu \mathrm{m}$; figs. 4, $9=30 \mu \mathrm{m}$; and figs. 1, 2, 6, 8, 10, $14=50 \mu \mathrm{m}$ ) 

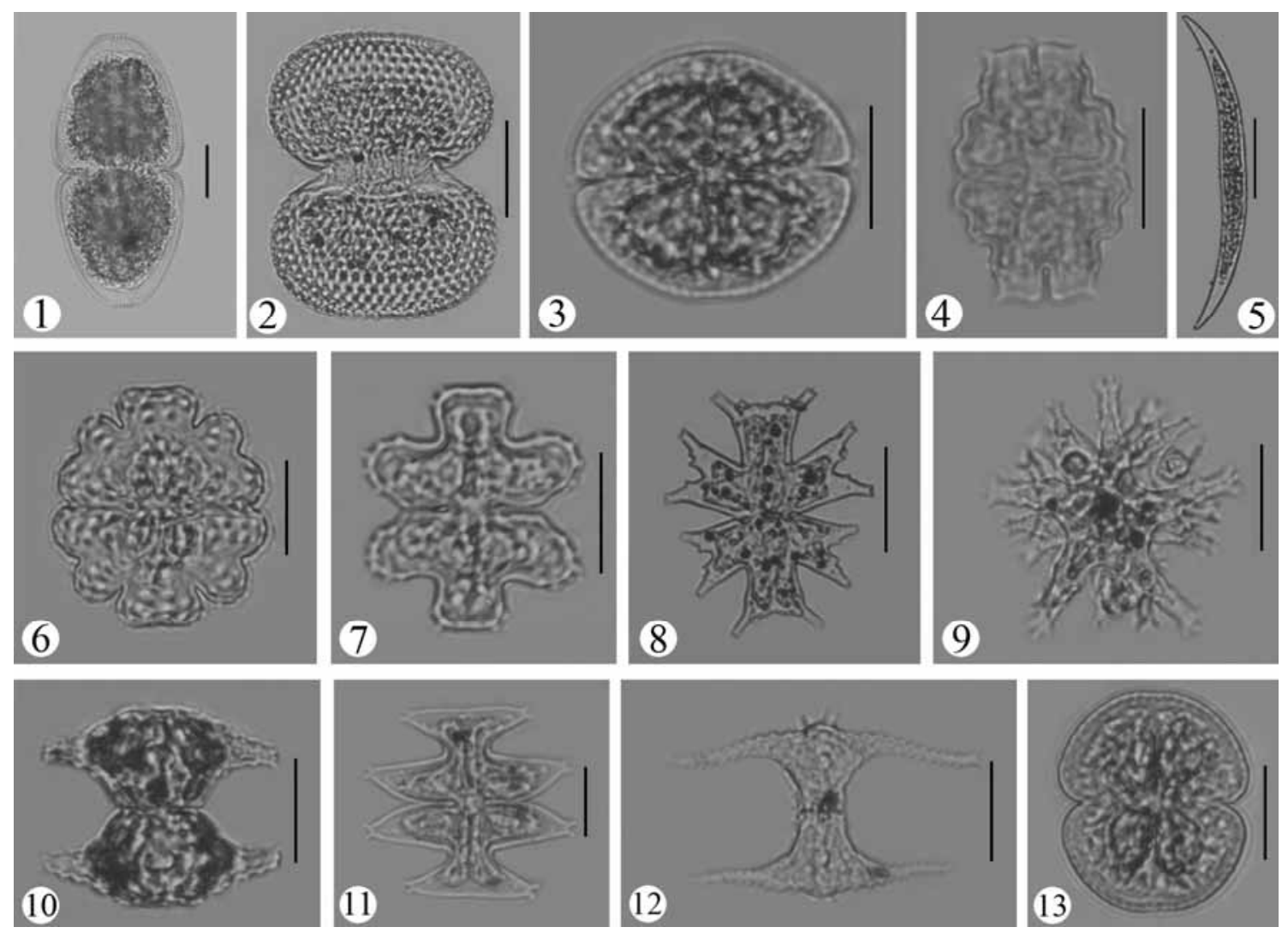

Plate 2. (Fig. 1. Cosmarium javanicum Nordst., Fig. 2. Cosmarium pardalis Cohn., Fig. 3. Cosmarium obsoletum (Hantz.) Reinsch, Fig. 4. Euastrum bidentatum Näg., Fig. 5. Closterium dianae Ehr. ex Ralfs var. dianae, Fig. 6. Euastrum spinulosum Delp., Fig. 7. Euastrum platycerum Reinsch, Fig. 8. Micrasterias mahabuleshwarensis Hobson, Fig. 9. Staurastrum sexangulare Lund. var. productum Nordst., Fig. 10. Staurastrum sexcostatum Bréb. ex Ralfs var. productum (W. West) G.S. West, Fig. 11. Micrasterias pinnatifida (Kütz.) Ralfs, Fig. 12. Staurastrum leptocladum Nordst. var. cornutum Wille, Fig. 13. Cosmarium lundelli Delp. var. ellipticum W. et G.S. West f. minus Prescott)

(Scales in the figs. $3,4,6,7,9,10,11,12,13=20 \mu \mathrm{m}$; figs. $1,2=30 \mu \mathrm{m}$; fig. $8=50 \mu \mathrm{m}$; and fig. $5=80 \mu \mathrm{m}$ )

\section{ACKNOWLEDGEMENT}

We are grateful to the Head, Department of Botany, Lucknow University, India for laboratory facility. We also acknowledge to the Director, National Botanical Research Institute, Lucknow for his concent to consult the library. Thanks to the Warden and Staffs of the Koshi Tappu Wildlife Reserve for their kind cooperation.

\section{REFERENCES}

Alfinito, S. and B. Fumanti. 1980. Contribution to the knowledge of fresh water algae from lake Hamun-i-puzak (Iran). Nova Hedwigia 33(14):873-882.

Bando, T., T. Nakano and M. Watanabe. 1989. The desmid flora of Kathmandu, Nepal. Bull. Natn. Sci. Mus., Ser. B. Tokyo, 15:1-25. 
Bharati, S.G. and G.R. Hegde. 1982. Desmids from Karnataka state and Goa, Part III. Genus Cosmarium Corda. Nova Hedwigia 36:733757.

Capdevielle, P. and A. Coute'. 1980. Quelques Staurastrum Meyen (Chlorophycées, Desmidiacées) rare ou nou veaux pour la France. Nova Hedwigia 33:859-882.

Flint, E.A. and D.B. Williamson. 1998. Desmids (Chlorophyta) in two ponds in Central Canterbury, New Zealand. Algological Studies 91:71-100.

Förster, K. 1965. Beitrag zur kenntais der Desmidiaceen-flora von Nepal. Erg. Forschunturn Nepal Himalaya. Khumbu Himal 1(2):25-58.

Gerrath, J.F. and D.M. John. 1988. The desmids of Ghana, West Africa-I. Nova Hedwigia 46(12):187-230.

Habib, I. and U.K. Chaturvedi. 1995. On some desmids from Nepal. J. Ind. Bot. Soc. 74:277282.

Habib, I. and U.K. Chaturvedi. 1997. Contribution to the knowledge of desmids from Nepal. Phykos 36:27-36.

Hickel, B. 1973. Limnological investigations in lakes of Pokhara valley, Nepal. Int. Rev. ges Hydrobiol 58(5):659-672.

Hirano, M. 1955. Fresh water algae. In: Fauna and Flora of Nepal Himalaya. (ed.) Kihara, H. Fauna and Flora Research Society, Kyoto Univ., Japan, pp. 5-42.

Hirano, M. 1963. Fresh water algae from the Nepal Himalaya, collected by a member of the Japanese Climbing Expedition. Contr. Biol. Lab., Kyoto Univ., Japan, 16:1-23.

Hirano, M. 1969. Fresh water algae from Langtang Himal, Nepal Himalaya. Contr. Biol. Lab., Kyoto Univ., Japan, 22:1-42.
Hirano, M. 1984. Fresh water algae from east Nepal. Study reported of Baika Junior College 32:197-215.

Ichimura, T. and F. Kasai. 1982. New mating groups, group $\mathrm{H}$ and group I of Closterium ehrenbergii Meneghini from Kathmandu valley and terai plains of Nepal. In: Reports on the Cryptogamic Study in Nepal. (ed.) Otani, Y. National Science Museum, Tokyo, Japan, pp. 61-73.

Kim, H.S. 1996. Desmid (Staurastrum and Staurodesmus) from Kyongsangnam-Do, Korea. Nova Hedwigia 62(3-4):521-541.

Kouwets, F.A.C. 1987. Desmids from the Auvergne (France). Hydrobiol 146:193-263.

Kusel-fetzmann, E. 1969. Einige Algen aus Nepal. Khumbu Himal 1(6):37-56.

Nakanishi, M. 1986. Limnological study in Phewa, Begnas and Rupa lakes. In: Studies on distribution, adaptation and evolution of microorganisms in Nepal Himalayas. (ed.) Ishida, Y. Ministry of Education, Science and Culture, Kyoto, Japan, pp. 3-13.

Nurul Islam, A.K.M. 1970. Contributions to the knowledge of desmids of East Pakistan, Part I. Nova Hedwigia 20:903-983.

Nurul Islam, A.K.M. and A.K. Yusuf Haroon 1980. Desmids of Bangladesh. Int. Rev. ges Hydrobiol 65(4):551-604.

Nurul Islam, A.K.M. and H.M. Irfanullah. 1999. New records of desmids for Bangladesh- III. 24 taxa. Bangladesh J. of Plant Taxon 6(2):91-104.

Prasad, B.N. and P.K. Misra. 1992. Fresh water algal flora of Andaman and Nicobar Islands. Vol. 2. B. Singh and M.P. Singh Publ., Dehradun, India, 284 p. 
Sahin, B. 2005. A preliminary checklist of desmids of Turkey. Crypt. Algol. 26(4):399-415.

Scott, A.M. and G.W. Prescott. 1961. Indonesian desmids. Hydrobiologia 17(1-2):1-132.

Shrestha, B. and J.D. Manandhar. 1983. Contribution to the algal flora of Kathmandu valley. J. Inst. Sci. Techn. 6:1-6.

Therezien, Y. 1985. Contribution a l'Etude des Algues d'Eau Douce de la Bolivie Les Desmidiales. Nova Hedwigia 41:505-576.

Tiffany, L.H. and M.E. Britton. 1952. The Algae of Illinois. Hafner Publ. Co. New York, 407 p.

Turner, W.B. 1892. The fresh water algae of east India. Kongl. Sv. Vet. Akademiens Handlingar 25(5):1-187.
Watanabe, M. 1982. Observation on the genus Closterium from Nepal. In: Reports on the Cryptogamic Study in Nepal. (compiled) Y. Otani. National Science Museum, Tokyo, Japan, pp. 47-59.

Watanabe, M. 1995. Algae from lake Rara and its vicinities, Nepal Himalayas. In: Cryptogams of the Himalayas, Vol. 3, Nepal and Pakistan. (eds.) Watanabe, M. and H. Hagiwara. National Science Museum, Tsukuba, Japan, pp. 1-17.

Yacubson, S. 1980. The phytoplankton of some fresh water bodies from Zulia state (Venezuela). Nova Hedwigia 33:279-339. 